\title{
The Psychological Effects of a lifestyle Management Course on War Veterans and Their Spouses
}

\author{
Grant J. Devilly \\ University of Melbourne
}

This research assessed the effect on a war-veteran outpatient group in a week-long residential lifestyle-management course. This course also included the veterans' partners, and all participants were assessed at intake, postintervention, and at three- and six-month follow-ups. In summary, it was found that while there was a statistically significant drop in PTSD symptomatology for the veterans, the clinical utility of this improvement was minimal, with an estimated effect size of $d=0.19$ by six-month follow-up. However, the veterans displayed a significant decrease in measures of depression, anxiety, and stress by six-month follow-up, all with small-tomoderate effect sizes. Likewise, ratings of anger showed statistically significant improvement with a moderate effect size. While dyadic adjustment displayed a significant improvement to six-month follow-up, the derived effect size was small for the veterans. The spouses (all females in this study) displayed larger effect sizes on all measures, with the exception of ratings of anger, where a small effect was noted. Subjective quality-of-life indices displayed a significant change in the desired direction, although with a minimal effect for the veterans and a small effect size for the females. It was not feasible to have a control group during this naturalistic investigation and, therefore, caution is advised in over-generalizing from these data. However, these results warrant further 'controlled' investigation into the inclusion of spouses in the treatment of veterans and the utility of lifestyle-management courses as a first step in the treatment of trauma related problems that have become chronic in nature within the veteran community. (c) 2002 Wiley Periodicals, Inc. J Clin Psychol 58: 1119-1134, 2002.

Keywords: veteran; lifestyle; trauma; psychopathology; caregiver; CBT; marital

The author would like to acknowledge and note the considerable input of the directors and staff of the Queensland Vietnam Veterans Counseling Services.

Correspondence concerning this article should be addressed to: Dr. Grant Devilly, Department of Criminology, University of Melbourne, Parkville, Victoria 3010, Australia; e-mail: dev@crim.unimelb.edu.au. 
With an aging Vietnam veteran population approaching retirement, some of the major challenges facing veteran services is the continued physical and mental health of the veteran and the well-being of the veteran's household. Many of the veterans have already received pensions relating to mental health problems, and with increased time spent in the company of their spouses during retirement it is becoming more important to include these spouses in intervention programs.

O'Toole et al. (1996) looked at 1,000 randomly selected Australian Vietnam veterans and found the lifetime prevalence rate of PTSD to be $20.9 \%$ (SCID-PTSD scale) with $11.6 \%$ of the sample experiencing current PTSD symptoms. A total of $59.6 \%$ met criteria for any diagnosis, with $33.1 \%$ of the sample reporting this within the last month. It has also been shown (Beckham, Lytle, \& Feldman, 1996) that the partners of Vietnam veterans with PTSD report increased "caregiver burden" and increased psychological distress, anxiety, and dysphoria commensurate with the veteran's symptom severity. It was further found that changes in burden "positively predicted individual changes in caregiver psychological distress, dysphoria, and state anxiety" (p. 1068) leading these authors to suggest that "because PTSD in Vietnam combat veterans appears to be both severe and chronic, interventions designed to help partners cope more effectively may be fruitful" (p. 1072).

Repeatedly, authors have emphasized the need to target the associated symptoms of PTSD in Vietnam veterans independently from the trauma treatments (e.g. Keane, Fairbank, Caddell, \& Zimmering, 1989; Cooper \& Clum, 1989; Frueh, Turner, \& Beidel, 1995; Frueh, Turner, Beidel, Mirabella, \& Jones, 1996; Johnson, Feldman, Southwick, \& Charney, 1994) and others have stressed the importance of the caregiver or spouse in this process and the reintegration of the veteran back into his former roles of father, husband, and breadwinner (Solomon, 1988; Beckham, Lytle, \& Feldman, 1996). However, until now no study has quantitatively assessed the utility of including spouses in the grouptreatment process, and none have assessed the efficacy of such programs on an outpatient basis. A study conducted within the Queensland Vietnam Veterans Counseling Service (VVCS), and funded by the Department of Veterans Affairs, has gone some way to address this and add to the descriptive essay on this topic by Erlanger and Fothergill (1998). This course, the lifestyle-management course, generally aims to maintain and where possible improve the quality of life for the participants. Specifically, the objectives of this course are to provide information on the principles of a healthier lifestyle, to enhance beliefs and attitudes that the person can be an agent of change in their own life, to provide strategies for creating a healthier personal lifestyle, and to promote skills in linking and relating to family and others. It should also be made clear that this course was not specifically designed for veterans or spouses with any particular presentation. In Australia veterans are also a notoriously difficult population to attract into treatment, preferring instead to isolate themselves and their family in geographically remote regions or maintaining an interpersonally restricted environment. It was also hoped that such a course would help demystify the counseling process and help initiate more specific treatment. This study assessed the efficacy of a lifestyle-management course (LM) which included both veterans and their spouses in the process.

Method

\section{Design}

This study used a naturalistic, non-randomized design with one condition where participants received a Lifestyle Management Course (LM). Longitudinal measures were collected 
at pre- and post-intervention, three-month follow-up (postal) and six-month follow-up (postal).

\section{Participants}

Two hundred and nine participants attended the LM, of which $111(53 \%)$ were male veterans and $98(47 \%)$ were non-veteran females. This course did not exclude female veterans, though none applied for attendance. Of the males who attended, two were single at intake, 91 were married, five were currently divorced, four were separated, and nine were in a de facto relationship. Of these males, 10 lived alone, one lived alone with his child, 41 lived with their spouse alone, 55 lived with their spouse and children, one lived with a friend, and three lived under some "other arrangement." Further, 103 of the males lived in a house, seven in an apartment, and one in a travel trailer. The average age of the males was 58.19 years $(\mathrm{sd}=4.13)$, the average age of the females was 48.38 years $(\mathrm{sd}=4.81)$, and the median (and modal) personal/household income (depending upon relevance to the participant) was within the AUS\$26,000 to \$40,999 range.

The participants were referred by contract counselors, center-based counselors, ex-service organizations, and allied health providers. Eligibility was very broad in that the criteria were: one of the participants must have held veteran status; only one course per couple was allowed; and a basic selection procedure applied whereby the counselor's recommendation of suitability for a group-based intervention held primary weight. While the course was designed primarily for couples, six single male veteran attendants were also permitted. There were 12 groups in total that were run in three primary locations: north, central, and southern Queensland, with the average waiting period for a lifestyle course of six months. All participants were advised that the questionnaire packages would remain confidential and also that any individuals' disclosures during the program should also be treated as confidential by all participants. Each cohort contained ten veterans.

\section{Measures}

Measures were selected due to their specificity in relation to individual sessions that were provided. For example, the Abbreviated Dyadic Adjustment Scale was administered to reflect changes that may have occurred following the groups that were run on communication styles and techniques. Severity of presenting complaints was assessed throughout the study using the following three measure:

Depression Anxiety Stress Scale 42 (DASS; Lovibond \& Lovibond, 1995). The DASS is a 42-item self-report inventory that yields three factors: depression, anxiety, and stress. This measure proposes that physical anxiety (fear symptomatology) and mental stress (nervous tension and nervous energy) factor out as two distinct domains. This screening and outcome measure reflects the past seven days. Gamma coefficients that represent the loading of each scale on the overall factor (total score) are .71 for depression, .86 for anxiety, and .88 for stress. Reliability of the three scales is considered adequate and test-retest reliability is likewise considered adequate with .71 for depression, .79 for anxiety and .81 for stress (Brown, Chorpita, Korotitsch, \& Barlow, 1997). Exploratory and confirmatory factor analyses have sustained the proposition of the three factors $(p<.05$; Brown et al., 1997). The DASS anxiety scale correlates .81 with the Beck Anxiety Inventory (BAI), and the DASS Depression scale correlates .74 with the Beck Depression Scale (BDI). 
Impact of Event Scale (IES; Horowitz, Wilner, \& Alvarez, 1979). The IES is a 15-item questionnaire evaluating experiences of avoidance and intrusion which attempts to "reflect the intensity of the post-traumatic phenomena" (McGuire, 1990). Both the intrusion and avoidance scales have displayed acceptable reliability (alpha of .79 and .82, respectively), and a split-half reliability for the whole scale of .86 (Horowitz et al., 1979). The IES has also displayed the ability to discriminate a variety of traumatized groups from non-traumatized groups (see Briere, 1997, for review). This measure was administered at pre- and post-intervention and at three- and six-month follow-ups.

Abbreviated Dyadic Adjustment Scale (ADAS; Sharpley \& Rogers, 1984). The ADAS is a seven-item scale derived from the 32-item Spanier Dyadic Adjustment Scale (Spanier, 1976). These items were suggested as useful in a screening test following the findings by Sharpley and Cross (1982) that six of the items in the full scale correctly classify $92 \%$ of cases with high or low marital satisfaction (and one item, "happiness," correctly classified $65 \%$ of cases on its own). Alpha reliability for the ADAS is .76, an item-total correlation of .57 or greater, and a range in inter-item correlations from .34 to .71 (and hence no redundant items are apparent) and, by comparing with married, separated, and divorced couples, the scale appears to be able to "differentiate between persons who were dissatisfied with their marriages according to external criteria and those who remained together" (Sharpley and Rogers, 1984). The major psychometric weakness of this scale is the lack of a test-retest reliability.

Comprehensive Quality of Life Inventory-Version 4 (COMQOL4; Cummins, 1994). The COMQOL is a 35 -item self-report questionnaire that utilizes both objective and subjective measures for quality of life within seven domains: Material Well-being; Health; Productivity; Intimacy; Safety; Place In Community; and Emotional Well-being. This instrument is based upon the propositions that: (a) Quality of life (QOL) is an aggregate of both objective (O) and subjective (S) components; (b) Each OQOL and SQOL component is composed of the above seven domains; (c) Each OQOL is obtained by taking an aggregate score for objective indices relevant to that domain; and (d) SQOL for a domain is a product of the perceived satisfaction with that domain weighted by its perceived importance to the individual. This screening and outcome measure reflects the patient's current situation and beliefs. Alpha for the subscale comprising the 21 individual objective items is .50, overall domain alpha for the Importance subscale of the subjective measures is .65 , and .73 for satisfaction. Test-Retest correlations for the subjective measure of Importance yielded a coefficient of .60 and for satisfaction a coefficient of .36. Overall, this measure displays acceptable test-retest reliability and internal consistency. To date, two domains have been tested for convergent validity. Health has been compared to the Nottingham Health Index, and Intimacy to the Personal Assessment of Intimacy in Relationships. Both have demonstrated acceptable validity. However, nomological validation of health satisfaction with the question "How do you feel about your health overall?" produced a correlation of .88. People with a psychiatric condition have displayed a lower life quality using this measure, particularly in the domain of intimacy (Cummins, 1997).

Novaco Anger Inventory-Short Form (NAI). The Novaco Anger Inventory-Short Form was adapted from the Long Form (Novaco, 1975) and contains 25 of the original 90 items. The NAI-Long Form-purports to measure the degree of provocation or anger people would feel if placed in certain situations. This scale displays a convergent validity of .46 with the Buss-Durke Hostility Inventory, and .41 with the Aggression subscale of 
the Personality Research Form (Huss, Leak, \& Davis, 1993) as well as a test-retest reliability of between .78 and .91 (Mills, Kroner, \& Forth, 1998). In order to ascertain the validity of the Short Form a factor analysis was applied to the current full data set at intake $(N=207)$ and four factors with an eigenvalue above 1.00 were derived. However, one factor derived an eigenvalue of 12.62 and accounted for over $50 \%$ of the variance. Only one item had a higher factor loading on any of the factors other than factor one. The factor loadings for factor one ranged between .53 and .79, with an average loading of .71 . It is suggested that this scale, therefore, reliably only consists of one factor (anger). A Cronbach's alpha of .96 was derived for the scale with an average inter-item correlation of .49, an item-total correlation of between .50 and .77, and a split-half reliability of .93 . No test-retest reliability is available for this test.

Alcohol Consumption Estimate (ACE). This questionnaire is a non-standardized questionnaire which asks how many drinks within the three alcohol categories (beer, wine, and spirits) the participant has on average each week. Therefore, this is a self-perception estimate of alcohol intake and is open to the lack of reliability common with this form of data procurement. However, the assessment on this construct necessarily needed to be quick and non-poignant to improve compliance with the overall assessment battery. Hence, a compromise that at least gave us self-reported alcohol intake was utilized. Subsequent research by the first author suggests that this method of assessment is reasonably valid, with 59 male veterans and 49 female spouses completing the ACE and the Alcohol Use Disorders Identification Test (AUDIT) and deriving a Spearman's rank correlation coefficient of .83 for the females and .82 for the males $(p<.0001)$.

Credibility/Expectancy Questionnaire (CEQ; Devilly \& Borkovec, 2000). The CEQ is self-report questionnaire which was developed for use by Borkovec and Costello (1993) and derives two factors: expectancy for change and treatment credibility (Devilly \& Borkovec, 2000). These factors have been found to be stable across different populations, with high internal consistency within each factor (standardized $\propto=0.84$ to 0.85 for the whole scale). Expectancy displays a one-week test-retest reliability of $r=0.82$, a credibility test-retest reliability of $r=0.75$, and a composite reliability of $r=0.83$. It has also been shown to differentiate between different treatment rationales and, while evidence is still being gathered, the expectancy factor displays some tendency to correlate with outcome (Devilly \& Borkovec, 2000). The CEQ was administered during the first day of the program, after the intervention rationale was given to the participants of the course, and the results were not disclosed to the therapist. For analyses, all items were standardized and composites were derived for the expectancy and credibility factors.

\section{Procedure}

The LM is essentially run for five consecutive days in a residential setting and includes groups on anger management, self-esteem issues, effective communication, PTSD information (particularly for the spouses), diet and nutrition, medical issues, alcohol and depression, problem solving and goal setting, relaxation and medication, and personal care among other activities.

An outline of the LM is provided in Table 1 . This is necessarily a basic overview, but this course is based upon CBT procedures, is multidisciplinary, and has a day and time structure. The CBT procedures include psycho-education, modeling and practice for groups such as the stress group, anger management and conflict resolution group, and relaxation 
Table 1

Example of Lifestyle Management Programme Outline

\begin{tabular}{llcl}
\hline \multicolumn{1}{c}{ Activity } & $\begin{array}{c}\text { Time } \\
\text { Date }\end{array}$ & \multicolumn{1}{c}{ Trainer } \\
\hline Day 1 & Welcome to the course & 30 & Staff \\
& Assessment & 60 & Psych/SW \\
& Getting to know each other & 60 & Staff \\
& Diet and nutrition & 90 & Nutritionist \\
& Relaxation and meditation & 90 & Psych/SW \\
Day 2 & Wake up and walk & & Staff \\
& Communication 1 & 30 & Psych/SW \\
& PTSD (psychoeducation) & 90 & Staff/Veteran \\
& Communication 2 & 180 & Psych/SW \\
& Taking care of yourself & 90 & Psych/SW/Contract \\
& Stress & 90 & Psych/SW \\
& Men's group and women's group & 90 & Staff \\
Day 3 & Wake up and walk & $30-120$ & Staff \\
& Medical issues/Medications & 30 & Psychiatrist/Pharmacist \\
& Alcohol and depression & $90-120$ & Psych/SW \\
& Excursion out of camp & 90 & Staff \\
Day 4 & Wake up and walk & $\frac{1}{2}$ day & Staff \\
& Anger management and conflict resolution & 30 & Psych/SW \\
& Self esteem & 90 & Psych/SW \\
& Communication 3 & 90 & Psych/SW \\
& Problem solving and goal setting & 90 & Psych/SW \\
& Discovering boundaries & 90 & Psych/SW \\
& Combined group & 90 & Staff \\
Day 5 & Wake up and walk & $30-120$ & Staff \\
& Open forum & 30 & All staff \\
& Post-Intervention Assessment & 60 & Staff $/ S W$ \\
& Going home \& photo time & 45 &
\end{tabular}

Note Staff $=$ Any or multiple members of staff; Psych $=$ Psychologist SW $=$ Social Worker Contract $=$ Contracted Counsellor.

and meditation group. The psycho-educational aspects are based upon the results of social, medical, and psychological research obtained in Australia and overseas, and exposure protocols are taught, modeled, and practiced during the week-long residential. An obvious example of this is even participating in the course for a group of veterans who tend to score highly on social phobia and fear of negative evaluation.

Those running the groups are either staff members of the Vietnam Veterans Counseling Service (usually three to four are present during the whole course) or contracted counselors who have specialized training in the group they run. The staff of the VVCS are all either psychologists or social workers, have a postgraduate qualification, and partake in peer supervision and continuing education. However, different VVCS centers have different individuals running the course and internal consistency between cohorts has not been assessed. Fidelity ratings could not be obtained due to the resistance by veterans to recording sessions. As mentioned, this was a naturalistic design and the introduction of audio or visual recordings would have interfered with the smooth running of the groups, 
changed the participant base, and it is doubted that such a practice would have been ethical in the normal practice of this federally funded service.

\section{Results}

Analyses were conducted to investigate the structure of the data and statistical significance of change over time. Further analyses were conducted to investigate the clinical relevance of the changes over time.

\section{Pre-Intervention}

Of the 209 participants (111 males, 98 females) who attended the LM course, 207 completed the post-intervention measures (110 males, 97 females), 165 completed the threemonth follow-up (86 males, 79 females) and 141 completed the six-month follow-up (74 males, 67 females). A 2 (sex; male and female) $\times 3$ (time point; pre-intervention, threemonth and six-month follow-up) chi-square test showed the questionnaire response rate between the sexes to be equivalent over time $\left(\chi^{2}(2)=.04, n s\right)$. Independent $t$-tests on pre-treatment outcome measures, marital status, living arrangements, rationale credibility, and treatment expectancy showed no significant difference between the presentation of those who dropped-out at six-month follow-up and those who completed their assessment packages, even without Bonferroni corrections $(p<.05)$.

As the 12 groups were run by three different VVCS centers (Townsville, Rockhampton, and, very predominantly, Brisbane) checks were made as to whether there was any difference in results with regard to which center provided the intervention. It was found that there was no significant difference $(p<.05)$. It should be noted, however, that the Rockhampton and Townsville centers accounted for less that $14 \%$ of the complete data sets for any one outcome measure.

Independent $t$-tests were applied to the intake data, with gender as the independent variable, to assess differences between these two distinct populations. A significant difference was found for depression $(t(205)=7.11, p<.0001)$; anxiety $(t(205)=7.31, p<$ $.0001)$; stress $(t(205)=9.22, p<.0001)$; anger $(t(205)=9.41, p<.0001)$; self-reported alcohol intake $(t(207)=5.79, p<.0001)$; objective quality of life $(t(199)=-9.00, p<$ $.0001)$; and subjective quality of life $(t(205)=-7.18, p<.0001)$, but not with dyadic adjustment $(t(204)=-.07, n s)$. For this reason gender was treated as an independent variable in the repeated measure analyses.

As can be seen in Table 2, at intake the male participants scored, on average, within the severe to extremely severe range on depression, anxiety, and stress, more than one standard deviation above a chronic PTSD sample on the Impact of Event Scale, to be significantly bothered by anger and to self-report the consumption of alcohol to the equivalent of 15 cans of full-strength beer a week (although this has a very high variance, ranging from 0 to the equivalent of $115375-\mathrm{ml}$ cans). These males reported to be generally satisfied regarding the relationship with their spouse. The female participants scored, on average, within the mild range on depression, anxiety, and stress, to be less irritable than their spouses and to self-report the consumption of alcohol to the equivalent of just over two cans of full strength beer a week (ranging from the equivalent of 0 to nearly 17 cans a week). The females reported a similar level of marital satisfaction to the males. It should also be noted that the males scored lower than the females on both subjective and objective quality of life, and that the male scores on the subjective quality of life suggest a very impaired and dysphoric sample. 
Table 2

Intake, Post-intervention, Three-Month and Six-Month Follow-up Participant Numbers, Means and Standard Deviations for Outcome Measures

\begin{tabular}{|c|c|c|c|c|c|c|}
\hline \multirow[b]{2}{*}{ Measure/Assessment Point } & \multicolumn{3}{|c|}{ Males } & \multicolumn{3}{|c|}{ Females } \\
\hline & $N$ & Mean (sd) & $d(\mathrm{P})$ & $N$ & Mean (sd) & $d(\mathrm{P})$ \\
\hline \multicolumn{7}{|l|}{ Depression (DASS 42) } \\
\hline Intake & 111 & $23.68(11.47)$ & - & 96 & $12.19(11.77)$ & - \\
\hline Post-Intervention & 109 & $12.36(9.91)$ & $0.94(0.99)$ & 94 & $5.81(7.66)$ & $0.61(0.99)$ \\
\hline 3-month Follow-up & 86 & $20.34(12.39)$ & $0.28(0.61)$ & 79 & $8.37(9.23)$ & $0.35(0.78)$ \\
\hline 6-month Follow-up & 74 & $19.97(13.47)$ & $0.30(0.63)$ & 66 & $8.02(9.52)$ & $0.38(0.76)$ \\
\hline \multicolumn{7}{|l|}{ Anxiety (DASS 42) } \\
\hline Intake & 111 & $19.62(10.33)$ & - & 96 & $9.42(9.63)$ & - \\
\hline Post-Intervention & 106 & $14.27(9.55)$ & $0.52(0.99)$ & 97 & $6.71(7.35)$ & $0.31(0.70)$ \\
\hline 3-month Follow-up & 86 & $16.59(9.99)$ & $0.30(0.66)$ & 78 & $6.01(7.63)$ & $0.38(0.80)$ \\
\hline 6-month Follow-up & 74 & $15.65(10.59)$ & $0.38(0.80)$ & 66 & $5.32(7.22)$ & $0.46(0.89)$ \\
\hline \multicolumn{7}{|l|}{ Stress (DASS 42) } \\
\hline Intake & 111 & $30.78(9.05)$ & - & 96 & $17.82(11.17)$ & - \\
\hline Post-Intervention & 109 & $19.87(10.62)$ & $0.97(0.99)$ & 97 & $9.57(8.86)$ & $0.76(0.99)$ \\
\hline 3-month Follow-up & 86 & $25.36(10.16)$ & $0.55(0.98)$ & 79 & $12.32(9.53)$ & $0.51(0.96)$ \\
\hline 6-month Follow-up & 74 & $26.31(10.81)$ & $0.45(0.91)$ & 67 & $11.78(9.72)$ & $0.55(0.96)$ \\
\hline \multicolumn{7}{|l|}{ Impact of Event Scale } \\
\hline Intake & 109 & $50.61(13.87)$ & - & 0 & - & - \\
\hline Post-Intervention & 107 & $42.72(17.71)$ & $0.48(0.97)$ & 0 & - & - \\
\hline 3-month Follow-up & 81 & 49.99 (14.79) & $0.04(0.09)$ & 0 & - & - \\
\hline 6-month Follow-up & 72 & $47.78(16.72)$ & $0.19(0.34)$ & 0 & - & - \\
\hline \multicolumn{7}{|l|}{ Abbreviated Dyadic } \\
\hline \multicolumn{7}{|l|}{ Adjustment Scale } \\
\hline Intake & 108 & $19.44(6.30)$ & - & 98 & $19.51(6.38)$ & - \\
\hline 3-month Follow-up & 82 & $20.10(6.21)$ & $0.11(0.18)$ & 75 & $21.03(5.16)$ & $0.26(0.51)$ \\
\hline 6-month Follow-up & 73 & $20.12(6.43)$ & $0.11(0.17)$ & 65 & 21.35 (5.99) & $0.29(0.57)$ \\
\hline \multicolumn{7}{|l|}{ Novaco Anger Inventory } \\
\hline Intake & 110 & 74.78 (13.99) & - & 97 & $51.58(21.14)$ & - \\
\hline 3-month Follow-up & 86 & $69.12(17.16)$ & $0.36(0.80)$ & 78 & $49.28(21.88)$ & $0.11(0.17)$ \\
\hline 6-month Follow-up & 75 & $66.72(18.20)$ & $0.50(0.95)$ & 67 & $46.12(19.06)$ & $0.27(0.51)$ \\
\hline \multicolumn{7}{|l|}{ Alcohol Intake } \\
\hline Intake & 111 & $267.38(381.97)$ & - & 98 & $40.94(66.41)$ & - \\
\hline 3-month Follow-up & 86 & $245.98(345.34)$ & $0.06(0.11)$ & 79 & $41.65(64.73)$ & $0.01(0.06)$ \\
\hline 6-month Follow-up & 76 & $218.97(318.53)$ & $0.14(0.23)$ & 67 & $45.27(74.55)$ & $0.06(0.11)$ \\
\hline \multicolumn{7}{|l|}{ COMQOL-T_-Objective } \\
\hline Intake & 106 & $58.13(8.87)$ & - & 95 & $69.00(8.17)$ & - \\
\hline 3-month Follow-up & 83 & $58.04(9.81)$ & $0.01(0.06)$ & 73 & $70.42(9.18)$ & $0.17(0.28)$ \\
\hline 6-month Follow-up & 74 & $57.35(10.52)$ & $0.03(0.07)$ & 67 & $69.70(9.34)$ & $0.08(0.13)$ \\
\hline \multicolumn{7}{|l|}{ COMQOL-T_-Subjective } \\
\hline Intake & 110 & $19.05(35.67)$ & - & 97 & $51.31(27.87)$ & - \\
\hline 3-month Follow-up & 84 & 23.15 (33.99) & $0.12(0.20)$ & 77 & $55.13(28.98)$ & $0.14(0.22)$ \\
\hline 6-month Follow-up & 74 & $23.57(36.87)$ & $0.13(0.21)$ & 66 & $59.56(25.64)$ & $0.30(0.60)$ \\
\hline
\end{tabular}

Note. $\mathrm{N}=$ number of participants at each assessment point; $\mathrm{sd}=$ standard deviation; $d=$ Cohen's effect size (from intake, for all participants, to the corresponding assessment point); $\mathrm{P}=$ power of effect size; DASS = Depression Anxiety Stress Scale; COMQOL-T $=$ Comprehensive Quality of Life Inventory-Total. 
Although this course did not specifically target veterans with PTSD, it is interesting to note that 99 males ( $91 \%$ of the male sample) scored above 33 on the Impact of Event Scale at intake, suggesting that this sample was predominantly disturbed by symptoms associated with the sequella to traumatic incidents.

\section{Repeated Measures Results}

Pre- to post-intervention measures, being only a week apart, are more likely to reflect satisfaction with the course as opposed to any meaningful change in functioning. Hence, these data were dealt with separately and not included in the repeated measures analysis. Therefore, the analysis that follows will be from intake through three-month follow-up to six-month follow-up. Effect sizes $\left(\eta^{2}\right.$; partial eta squared) and Power $(P)$ are reported after each analysis. Effect sizes from pre-intervention to the various time points for each measure (Cohen's $d$ ) and the associated Power $(P)$ are presented in Table 2 for both males and females. A 3 (Time) $\times 2$ (Gender) repeated measures MANOVA ${ }^{1}$ was applied to depression, anxiety, and stress, as measured by the DASS 42, from intake through three-month follow-up to six-month follow-up. This displayed a significant effect for Gender $\left(\right.$ Wilks' $\left.\Lambda(3,122)=.59, p<.0001, \eta^{2}=.41, P=0.99\right)$, with females scoring lower than the males at all time points, and a significant decrease in scores over Time $\left(\right.$ Wilks' $\left.\Lambda(6,119)=.68, p<.0001, \eta^{2}=.32, P=0.99\right)$. However, there appeared no effect for the interaction of Gender over Time (Wilks' $\Lambda(6,119)=.96, n s)$, suggesting that the patterns of change for the veterans and their partners were similar over the six months. Univariate analyses displayed depression, anxiety, and stress to all significantly decrease over time (all $p<.0001$ with a power of 0.99 ).

Turning to repeated measure ANOVAs, the Abbreviated Dyadic Adjustment Scale did not show a significant effect for Gender $(F(1,121)=.35, n s)$, but did show a very small trend toward significance for Time, although it should be noted that this is more a product of the large sample, as displayed by the very small effect, $(F(2,242)=2.71, p<$ $\left..07, \eta^{2}=.02, P=.53\right)$. There was also no significant interaction effect between the sexes over Time $(F(2,242)=1.66, n s)$.

The Novaco Anger Inventory displayed a significant effect for Gender $(F(1,127)=$ 63.82, $\left.p<.0001, \eta^{2}=.33, P=0.99\right)$, and although there was a highly significant effect over Time, this again is due to the large sample, as displayed by the small effect size, $\left(F(2,254)=13.88, p<.0001, \eta^{2}=.10, P=0.99\right)$. The interaction of Gender over Time was not significant $(F(2,254)=1.72, n s)$.

The Impact of Event Scale, which was administered to the veterans, displayed a trend for statistical significance over Time, but with a very small effect size $(F(2,124)=$ 2.86, $\left.p<.07, \eta^{2}=.04, P=0.55\right)$. Self-reported alcohol consumption displayed a significant effect for Gender $\left(F(1,130)=22.35, p<.0001, \eta^{2}=.15, P=0.99\right)$, but no significant effect for Time $(F(2,260)=1.26, n s)$, or for the interaction of Gender over Time $(F(2,260)=1.04, n s)$.

As can be seen from Figure 1, looking at the objective quality of life measures (COMQOL 4), there appeared a significant effect for Gender, with females scoring higher (Wilks' $\left.\Lambda(7,118)=.42, p<.0001, \eta^{2}=.58, P=0.99\right)$ and a small effect for Time (Wilks' $\left.\Lambda(14,111)=.82, p<.05, \eta^{2}=.18, P=.89\right)$, but not for the interaction of these two $($ Wilks' $\Lambda(14,111)=.92, n s)$. Subjective quality of life domains yet again displayed

\footnotetext{
${ }^{1}$ Using pre-treatment scores as covariates and conducting a MANCOVA for the DASS 42 and ANCOVAs for the other repeated measures analyses did not affect Time or the interaction effect of Gender over Time.
} 

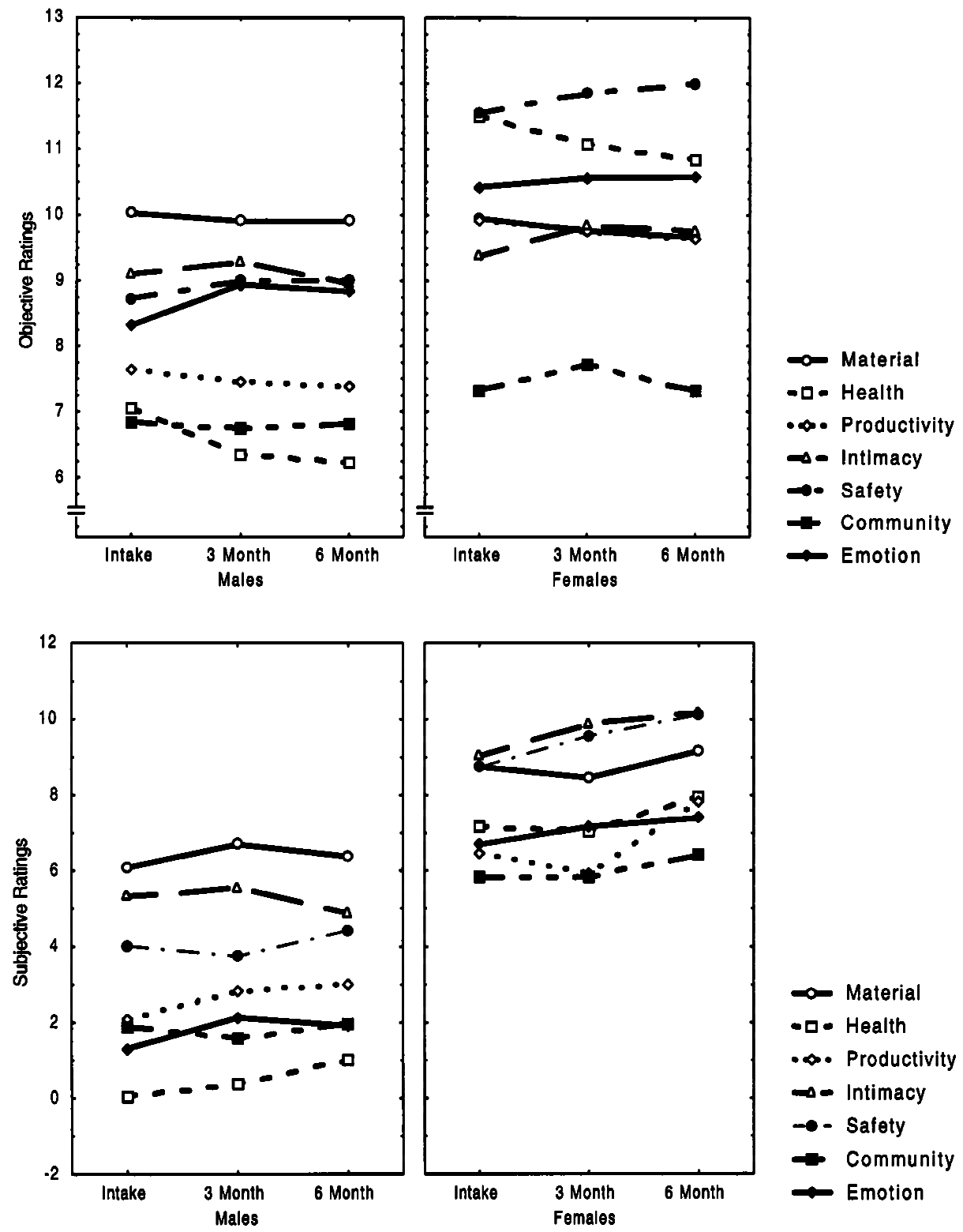

Figure 1. Mean scores over time, between male veterans and their female spouses on objective and subjective ratings for quality of life domains (COMQOL 4).

an effect for Gender, with females rating higher than the males $\left(W_{i l k s}^{\prime} \Lambda(7,119)=.70\right.$, $\left.p<.0001, \eta^{2}=.30, P=0.99\right)$, yet no significant effect for Time $($ Wilks' $\Lambda(14,112)=$ $.87, n s)$, nor an interaction effect $($ Wilks' $\Lambda(14,112)=.93, n s)$.

\section{Post-Hoc Comparisons}

Scheffé tests confirmed that the decrease in scores on depression, anxiety, and stress were significant between intake and three-month follow-up and between intake and six-month 
follow-up ( $p<.0001$ ), although not between three-month and six-month follow-ups. This same pattern also emerged for ratings of anger $(p<.001)$ and suggests that improvements were maintained over time on these dimensions.

\section{Pre- to Post-Intervention}

It is believed that the post-intervention assessment would be more indicative of satisfaction with the course than any long-term change in level of functioning and would also be an indirect measure of the distress caused by the content of the week-long course. Indeed when a 2 (Time) $\times 2$ (Gender) repeated measures MANOVA was applied to depression, anxiety, and stress, as measured by the DASS 42 there was a large effect for Time with both males and females displaying a decrease on all measures (Wilks' $\Lambda(3,194)=.42$, $\left.p<.0001, \eta^{2}=.58, P=0.99\right)$ and a significant effect for Gender with males scoring higher $\left(\right.$ Wilks' $\left.\Lambda(3,194)=.68, p<.0001, \eta^{2}=.32, P=0.99\right)$, and a significant interaction between the Genders over Time (Wilks' $\Lambda(3,194)=.92, p<.002, \eta^{2}=.08, P=$ .94). However, it should be noted that this interaction, while statistically significant, is hardly meaningful with such a small effect size. A dependent $t$-test displayed the veterans to decrease their ratings on the Impact of Event Scale by post-intervention $(t(104)=$ $4.35, p<.0001, d=.42, P=.92)$. These results suggest that this course did not aggravate the veterans' trauma-related symptoms and was not a stressful occasion for either the veterans or their spouses.

\section{Reliable and Clinical Change}

Although statistical significance may show a main effect for Time, this may not translate into a meaningful change clinically, when the reliability of the instrument is taken into account (Jacobson \& Truax, 1991). In order to judge whether individuals displayed improvement, a reliable change index was developed to measure those who improved or deteriorated $(p<.05)$ over time. The methods used for these analyses were those suggested by Jacobson and Truax (1991). Furthermore, in addition to reliable change, it is of interest to the clinician and of functional importance to the participant whether a change in classification occurs following intervention. For example, a participant that was severely depressed may have reliably decreased their depression rating and also now be only moderately or mildly depressed. Therefore, analyses were conducted that looked at which male and female participants reliably decreased or increased on various measures and also which participants reliably decreased or increased and changed classification. Male and female reliable change indexes were computed independently of each other, due to the difference between these two conditions at intake, and the pre-intervention standard deviation of each sample was used in the derivation of the reliable change index. These results are presented in Table 3. It should be noted that only measures were analyzed in this fashion for which a test-retest reliability coefficient was known, and hence the NAI (short form) and the ADAS were not investigated in this manner.

As can be seen from Table 3, with the DASS displaying a test-retest reliability of .71 for depression, .79 for anxiety and .81 for stress (Brown et al., 1997), the male standard error of the difference between any two assessment points was 8.74 for depression, 6.70 for anxiety, and 5.58 for stress. Change in status was derived by seeing which participants had reliably increased in functioning, according to the above description, and were also, at intake, two standard deviations above a normal sample but were below this at the respective follow-up time points. Change in status was also derived for those who had 
Table 3

Percentage of Participants Who Reliably Improved/Deteriorated (95\% Confidence) and Those Who Changed Clinical Status in a Positive or Negative Direction from Intake to the Follow-ups

\begin{tabular}{|c|c|c|c|c|}
\hline \multirow[b]{2}{*}{ Measure/Assessment } & \multicolumn{2}{|c|}{ Males } & \multicolumn{2}{|c|}{ Females } \\
\hline & $\begin{array}{l}\text { Reliable } \\
\text { Change }\end{array}$ & $\begin{array}{l}\text { Change } \\
\text { in Status }\end{array}$ & $\begin{array}{l}\text { Reliable } \\
\text { Change }\end{array}$ & $\begin{array}{l}\text { Change } \\
\text { in Status }\end{array}$ \\
\hline Depression (DASS 42) & $\left(\mathrm{S}_{\mathrm{diff}}=8.74\right)$ & $($ Critical $=20.57)$ & $\left(\mathrm{S}_{\mathrm{diff}}=8.92\right)$ & $($ Critical $=19.98)$ \\
\hline 3-month Follow-up & $11.63^{+}, 0.00^{-}$ & $11.63^{+}, 0.00^{-}$ & $10.13^{+}, 3.80^{-}$ & $10.13^{+}, 3.80^{-}$ \\
\hline 6-month Follow-up & $12.16^{+}, 1.35^{-}$ & $12.16^{+}, 1.35^{-}$ & $9.09^{+}, 1.52^{-}$ & $9.09^{+}, 1.52^{-}$ \\
\hline Anxiety (DASS 42) & $\left(\mathrm{S}_{\mathrm{diff}}=6.70\right)$ & $($ Critical $=14.20)$ & $\left(\mathrm{S}_{\mathrm{diff}}=6.24\right)$ & $($ Critical $=14.86)$ \\
\hline 3-month Follow-up & $15.12^{+}, 0.00^{-}$ & $9.30^{+}, 0.00^{-}$ & $10.26^{+}, 3.85^{-}$ & $8.97^{+}, 1.28^{-}$ \\
\hline 6-month Follow-up & $10.81^{+}, 5.41^{-}$ & $9.46^{+}, 2.70^{-}$ & $13.64^{+}, 0.00^{-}$ & $10.61^{+}, 0.00^{-}$ \\
\hline Stress (DASS 42) & $\left(\mathrm{S}_{\mathrm{diff}}=5.58\right)$ & $($ Critical $=25.25)$ & $\left(\mathrm{S}_{\mathrm{diff}}=4.87\right)$ & $($ Critical $=26.61)$ \\
\hline 3-month Follow-up & $32.56^{+}, 0.00^{-}$ & $25.58^{+}, 0.00^{-}$ & $18.99^{+}, 2.53^{-}$ & $12.66^{+}, 1.27^{-}$ \\
\hline 6-month Follow-up & $24.32^{+}, 1.35^{-}$ & $13.51^{+}, 0.00^{-}$ & $22.39^{+}, 1.49^{-}$ & $11.94^{+}, 1.49^{-}$ \\
\hline Impact of Event Scale & $\left(\mathrm{S}_{\mathrm{diff}}=7.07\right)$ & $($ Critical $=40.50)$ & - & - \\
\hline 3-month Follow-up & $22.79^{+}, 10.13^{-}$ & $7.60^{+}, 3.80^{-}$ & - & - \\
\hline 6-month Follow-up & $22.54^{+}, 9.86^{-}$ & $11.27^{+}, 5.35^{-}$ & - & - \\
\hline COMQOL-T_-Objective & $\left(\mathrm{S}_{\mathrm{diff}}=4.69\right)$ & $($ Critical $=60.11)$ & $\left(\mathrm{S}_{\mathrm{diff}}=4.32\right)$ & $($ Critical $=60.11)$ \\
\hline 3-month Follow-up & $11.39^{+}, \quad 6.32^{-}$ & $10.13^{+}, 2.53^{-}$ & $8.33^{+}, 4.17^{-}$ & $2.78^{+}, 2.78^{-}$ \\
\hline 6-month Follow-up & $9.59^{+}, 10.96^{-}$ & $8.22^{+}, 4.11^{-}$ & $13.43^{+}, 11.94^{-}$ & $5.97^{+}, 4.48^{-}$ \\
\hline COMQOL-T_-Subjective & $\left(S_{\text {diff }}=20.18\right)$ & $($ Critical $=1.39 *)$ & $\left(S_{\text {diff }}=15.77\right)$ & $($ Critical $=1.39 *)$ \\
\hline 3-month Follow-up & $15.66^{+}, 8.43^{-}$ & $13.25^{+}, 8.43^{-}$ & $15.79^{+}, 5.26^{-}$ & $2.78^{+}, 1.39^{-}$ \\
\hline 6-month Follow-up & $9.59^{+}, 9.59^{-}$ & $9.59^{+}, 6.85^{-}$ & $10.61^{+}, 1.52^{-}$ & $0.00^{+}, 0.00^{-}$ \\
\hline
\end{tabular}

Note. DASS42 = Depression Anxiety Stress Scale; COMQOL-T = Comprehensive Quality of Life Inventory Total; $\mathrm{S}_{\text {diff }}=$ Standard error of the difference in calculation of the reliable change index; Critical = critical score ( 2 standard deviations above a normal sample) used as change of status index $;^{+}=$improvement; ${ }^{-}=$deterioration; $*=$ Critical score for subjective COMQOL was based on the mean score per domain and standard deviation, as these figures were available.

reliably deteriorated, by looking at the percentage of participants who at intake were below the two standard deviation cut-off, but were above this at follow-up. For example, at six-month follow-up $24.32 \%$ of the males had reliably improved on the stress subscale of the DASS and $1.35 \%$ had reliably deteriorated. However, $13.51 \%$ of the males had reliably improved and changed status from being more than two standard deviations above a normal sample (i.e. scored greater than 25.25) at intake to being under this score by six-month follow-up. Also no males changed status due to deterioration of their functioning on this measure by six-month follow-up, although $1.49 \%$ of females did.

Of note in Table 3 is the much larger number of participants who had reliably improved compared to those who deteriorated at all time points and on nearly all symptomatic constructs. The only exception to this was with the objective measure of the Comprehensive Quality of Life Scale (COMQOL) for males. By six-month follow-up $10.96 \%$ of males had reliably deteriorated compared to $9.59 \%$ who had reliably improved. Although this difference is small it is nonetheless worthy of explanation. The second domain, as measured by the COMQOL, is that of health. The objective measure of this domain quantifies visits to the doctor, disabilities and/or medical conditions, and regular medication intake over the past three months. As can be seen from Figure 1, this domain shows a worsening for the males over time and, in fact, was the only statistically significant 
deterioration $(t(73)=2.14, p<.04)$ for this gender-based sample. However, this is not unexpected, as many of the veterans increased the number of visits to the doctor, were diagnosed with disabilities/medical conditions and increased their medication intake as a consequence of being involved with the Vietnam Veterans Counseling Service and being referred to various medical/psychological consultants. This should not be taken as a poor outcome for a quality of life index, but rather one of the processes necessary to eventually increase participants' quality of life, a point borne out by the increase within this domain on the subjective scores.

The largest impact on any of the measures was with stress. By six-month follow-up $13.51 \%$ of the males and $11.94 \%$ of the females had reliably improved and had also changed from being more than two standard deviations above a normal sample to being within the two standard deviations. The next highest for males was with depression, followed by the Impact of Event Scale. For females the next highest was anxiety followed by depression. It would also appear that for females the measure least affected was subjective quality of life.

\section{Qualitative Results}

At the end of each lifestyle course the participants were given the opportunity, on the post-intervention questionnaire battery, to write comments on the program. Nearly all comments were positive, with only one negative piece of feedback. The positive comments by the males centered mainly around the "sensitive, non-patronizing" way in which the facilitators dealt with issues, such as the Vietnam experience, and the overall informative content of the course. The females' most frequent comments were "I wish this had been available years ago" and comments relating to having been included in the intervention. The negative comments related to two veterans who had displayed animosity towards each other and the locking of a refrigerator on a lifestyle course that did not allow alcohol consumption. Both of these points were taken into account when planning the subsequent lifestyle course.

\section{Discussion}

This research sought to evaluate the psychological impact of a lifestyle-management program on veterans and their spouses. The results indicated that both veterans and their spouses displayed a significant decrease in measures of depression, anxiety, and stress by six-month follow-up, with moderate effect sizes on average. Likewise, ratings of anger showed statistically significant improvement for both males and females with a moderate effect size. While dyadic adjustment (marital happiness in this study) displayed a small improvement to six-month follow-up, the derived effect size was small. However, it is suggested that PTSD symptoms directly affect dyadic adjustment for the primary caregiver (Beckham et al., 1996) and so this is not a surprising event. To improve functioning within this domain one may also need to treat the PTSD that impacts this construct. However, it should also be noted that, on the whole, this population reported being within the higher satisfaction category of dyadic adjustment anyway. Also, while there was a statistically significant drop in PTSD symptomatology $(p<.001$; as measured by the Impact of Event Scale) the clinical utility of this improvement was small, with an estimated effect size of about $d=0.19$ by six-month follow-up. Likewise, Objective quality of life ratings (as measured by the seven domains of the COMQOL4) displayed improvement over time to six-month follow-up, although this had a small effect size. It should be 
noted that from the males' perspective objective and subjective quality of life was really quite low at all time points. Cummins (in press) has suggested that objective and subjective indicators are normally quite independent, with subjective QOL normally held within a small range under homeostatic control, but as objective quality of life becomes very low a threshold of living is reached and the objective and subjective indices begin to co-vary. Alcohol consumption was reasonably unaffected by the course for both males and females and may reflect the need for alcohol-specific treatment targeting at a later date with some individuals (the standard deviation of alcohol consumption within the male group was almost $30 \%$ greater than the mean alcohol consumption). It is suggested that to include more alcohol-related classes on the lifestyle course may be counter-productive and inappropriate for the many veterans and spouses for whom alcohol intake is not a presenting problem.

These results are broadly congruent with the findings of Beckham et al. (1996) and the suggestions of Keane et al. (1989), among others. However, while some have argued that under certain circumstances benefit can be obtained from the careful, conservative evaluation of studies without controls (Cummings \& Weiss, 1998), a controlled clinical trial is still imperative before a confident interpretation of these results can be made. This research utilized a naturalistic design where participants applied to be included in the course. It is equally possible that the improvement noted could be due to a general increase in contact with the Vietnam Veterans Counseling Service (VVCS), the increased use of health facilities and referrals, and/or the development of a peer-support network among the participants from having been together as a group, as opposed to the impact of the course content. Clarification of this point would need a control group who were on a waiting list to attend the lifestyle course and who had the same access to VVCS facilities as those who are in the intervention group. Furthermore, with the qualitative reports taken into account, one must stress the therapist process and non-specific variables that may affect the success of such courses. A controlled study will need to monitor consistent treatment delivery and check the quality of group content delivery. This was not possible in the current research.

This lifestyle course was not diagnostically specific in its aims and was offered to increase a sense of support within the veteran household, to highlight areas of concern within this setting, and to offer strategies and methods of dealing with common individual and interpersonal issues. However, with a mild to moderate effect on both veterans and their wives in areas of anxiety, stress, depression, and anger, with a very high power level, it is suggested that this course may act as a good initiation to the treatment of the traumatized veteran. Traditionally PTSD has been treated within a social vacuum, as a discrete entity conceptualized as being uninfluenced by environmental contingencies and social support. This program, however, could be the initiation of a more holistic approach to treatment. It may be that the lifestyle course helps prepare veterans and their partners for more specific treatment at a later stage. It would be particularly useful to trial the lifestyle course on its own against a wait-list control and a condition which entailed attendance at the lifestyle course followed by individualized treatment of the posttraumatic array via a validated system (e.g. Devilly \& Spence, 1999; Foa et al., 1991). This author has applied for funds to conduct such a research project. Such a study would also allow for randomization, fidelity checking, and a more in-depth collection of information regarding the veterans personal, combat-related, and therapeutic history, disability status, change in this status over time, and a more in-depth pursuit as to who dropped out and why.

The results of this initial study suggest that providing coping strategies, information, and methods of interaction to veterans and their spouses has a moderate effect size on 
indices of depression, anxiety, stress, and irritability and may act as a method for participants to move away from the preoccupation with the damaged self common in chronic trauma victims and towards a more sharing, outwardly-focused style of couple and family interaction. Controlled research is imperative.

\section{References}

Beckham, J.C., Lytle, B.L., \& Feldman, M.E. (1996). Caregiver burden in partners of Vietnam War veterans with Posttraumatic stress disorder. Journal of Consulting and Clinical Psychology, 64, 1068-1072.

Borkovec, T.D., \& Costello, E. (1993). Efficacy of applied relaxation \& cognitive-behavioral therapy in the treatment of generalized anxiety disorder. Journal of Consulting \& Clinical Psychology, 61, 611-619.

Briere, J. (1997). Psychological assessment of adult posttraumatic states. Washington, DC: American Psychological Association.

Brown, T.A., Chorpita, B.F., Korotitsch, W., \& Barlow, D.H. (1997). Psychometric properties of the Depression Anxiety Stress Scales (DASS) in clinical samples. Behaviour Research \& Therapy, 35, 79-89.

Cooper, N.A., \& Clum, G.A. (1989). Imaginal flooding as a supplementary treatment for PTSD in combat veterans: A controlled study. Behavior Therapy, 20, 281-391.

Cummins, R. (1994). The comprehensive quality of life scale (ComQol) [4th ed.]. Melbourne: School of Psychology, Deakin University.

Cummins, R. (1997). The comprehensive quality of life scale (ComQol) [5th ed.]. Melbourne: School of Psychology, Deakin University.

Cummins, R. (in press). Objective \& subjective quality of life: An interactive model. Social Indicators Research.

Cummings, P., \& Weiss, N.S. (1998). Case series \& exposure series: The role of studies without controls in providing information about the etiology of injury or disease. Injury Prevention, 4, $54-57$.

Devilly, G.J., \& Borkovec, T.D. (2000). Psychometric properties of the credibility/expectancy questionnaire. Journal of Behavior Therapy \& Experimental Psychiatry, 31, 73-86.

Devilly, G.J., \& Spence, S.H. (1999). The relative efficacy \& treatment distress of EMDR \& a cognitive behavior Trauma Treatment Protocol in the amelioration of posttraumatic stress disorder. Journal of Anxiety Disorders, 13 (1-2), 131-157.

Erlanger, H., \& Fothergill, N. (1998). From just coping to better living. Psychotherapy in Australia, $4,17-23$.

Foa, E.B., Rothbaum, B.O., Riggs, D.S., \& Murdoch, T.B. (1991). Treatment of posttraumatic stress disorder in rape victims: A comparison between cognitive-behavioral procedures and counseling. Journal of Consulting and Clinical Psychology, 59(5), 715-723.

Frueh, B.C., Turner, S.M., \& Beidel, D.C. (1995). Exposure therapy for combat-related PTSD: A critical review. Clinical Psychology Review, 15, 799-817.

Frueh, B.C., Turner, S.M., Beidel, D.C., Mirabella, R.F., \& Jones, W.J. (1996). Behaviour Research and Therapy, 34, 533-543.

Horowitz, M., Wilner, M., \& Alvarez, W. (1979). Impact of event scale: A measure of subjective stress. Psychosomatic Medicine, 41, 209-218.

Huss, M.T., Leak, G.K., \& Davis, S.F. (1993). A validation study of the Novaco Anger Inventory. Bulletin of the Psychonomic Society, 31, 279-281.

Jacobson, N.S., \& Truax, P. (1991). Clinical significance: A statistical approach to defining meaningful change in psychotherapy research. Journal of Consulting and Clinical Psychology, 59, $12-19$. 
Johnson, D.R., Feldman, S.C., Southwick, S.M., \& Charney, D.S. (1994). The concept of the second generation program in the treatment of post-traumatic stress disorder among Vietnam veterans. Journal of Traumatic Stress, 7, 217-235.

Keane, T.M., Fairbank, J.A., Caddell, J.M., \& Zimmering, R.T. (1989). Implosive (flooding) therapy reduces symptoms of PTSD in Vietnam combat veterans. Behavior Therapy, 20, 245-260.

Lovibond, P.F., \& Lovibond, S.H. (1995). The structure of negative emotional states: Comparison of the Depression Anxiety Stress Scales (DASS) with the Beck Depression and Anxiety Inventories. Behaviour Research \& Therapy, 33, 335-343.

McGuire, B. (1990). Post-traumatic stress disorder: A review. The Irish Journal of Psychology, 11, $1-23$.

Mills, J.F., Kroner, D.G., \& Forth, A.E. (1998). Novaco anger scale: Reliability and validity within an adult criminal sample. Assessment, 5, 237-248.

Novaco, R.W. (1975). Anger control: The development of an experimental treatment. Lexington, KY: Lexington.

O’Toole, B.I.O., Marshall, R.P., Grayson, D.A., Schureck, R.J., Dobson, M., French, M. et al. (1996). The Australian Vietnam veterans Health study: III. Psychological health of Australian Vietnam veterans and its relationship to combat. International Journal of Epidemiology, 25, 331-339.

Sharpley, C.F., \& Cross, D.G. (1982). A psychometric evaluation of the Spanier Dyadic Adjustment Scale. Journal of Marriage \& the Family, 44, 739-741.

Sharpley, C.F., \& Rogers, H.J. (1984). Preliminary validation of the Abbreviated Spanier Dyadic Adjustment Scale: Some psychometric data regarding a screening test of marital adjustment. Educational and Psychological Measurement, 44, 1045-1049.

Solomon, Z. (1988). The effects of combat-related post-traumatic stress disorder on the family. Psychiatry, 51, 323-329.

Spanier, G.B. (1976). Measuring dyadic adjustment: New scales for assessing the quality of marriage and similar dyads. Journal of Marriage and the Family, 38, 15-38. 